\title{
WORK HARD, PARTY HARD: HARM REDUCTION IN A POST-SECONDARY SETTING
}

\author{
VICTORIA FORD \\ MCGLLL UNIVERSITY
}

\author{
ALYSSA WOOSTER \\ MCGILL UNIVERSITY
}

\author{
MARY BARTRAM \\ MCGILL UNIVERSITY
}

\begin{abstract}
In the context of the increasing focus on harms, psychological safety, and mental health in post-secondary settings, this qualitative study explores the challenges and opportunities for harm reduction through focus groups with student leaders, service providers, and administrators in one large Canadian university. Key themes explored by participants include a pervasive work hard, party culture, clashes regarding how to define and operationalize harm reduction, broad approaches to harm reduction in tension with the risk of becoming a band-aid solution, and knowledge transfer and privilege in an academic context. These findings suggest possible avenues for harm reduction that could be implemented as part of the new post-secondary standard, as well as in society as a whole.
\end{abstract}

Keywords: harm reduction, mental health, post-secondary, Canada

\section{Résumé}

Dans le contexte de l'attention accrue à l'égard des méfaits, de la sécurité psychologique et de la santé mentale dans le milieu postsecondaire, cette étude qualitative explore les défis et les opportunités liés à la réduction des méfaits au travers de groupes de discussion composés de leaders étudiants, de fournisseurs de services et d'administrateurs au sein d'une grande université canadienne. Les thèmes clés qu'explorent les participants comprennent la culture répandue du travail acharné et de la fête; les disputes portant sur la définition et l'exécution de la réduction des méfaits; la tension entre les approches générales de réduction des méfaits et le risque d'appliquer des solutions de fortune; et le transfert des connaissances et le privilège dans le milieu universitaire. Ces résultats suggèrent des voies possibles pour la réduction des méfaits qui pourraient être suivies dans le cadre de la nouvelle norme postsecondaire, ainsi que dans la société en général.

Mots-clés : réduction des méfaits, santé mentale, postsecondaire, Canada

\section{Introduction}

With ample opportunity for harm in post-secondary settings, what is the potential role of harm reduction? Post-secondary education brings many beneficial academic and life experiences but can also introduce many potential risk factors: academic stress, new social settings, lack of parental supervision, and easier access to drugs and alcohol. Over the past decade, post-secondary institutions in Canada have confronted serious harms such as binge drinking at campus events, completed suicides on campus, and rape culture. Alarming increases in mental health problems have also been reported by post-secondary students, along with reports of surging demand for services. In response, post-secondary institutions are implementing everything from sexual violence prevention policies to orientation week changes to broad mental health strategies that include increases in mental health promotion activities and clinical services. At the national level, work is underway on a Psychological Health and Safety Standard for Post-Secondary Students (Mental Health Commission of Canada, 2019). In the context of this increasing focus on harms, psychological safety, and mental health, this qualitative study explores the challenges and opportunities for 
harm reduction in post-secondary settings through focus groups with student leaders, service providers, and administrators in one large Canadian university.

Harm reduction generally refers to efforts to reduce the negative impacts from the use of illegal drugs and other substances without requiring abstinence, and is increasingly being applied to a broader range of health behaviours (Hawk et al., 2017; Government of Canada \& Health Canada, 2018). Harm reduction has roots in activism and pragmatism. From its activist roots, harm reduction encompasses an "aggressively value neutral" stance adopted by activists (Klein, 2015, p. 465). For example, the Vancouver Area Network of Drug Users' (VANDU) Manifesto calls for:

...the right to obtain, prepare, and ingest drugs, and to be intoxicated on drugs, according to our own personal decisions without criminalization or unsought interference from other individuals or organizations, as long as our drug use does not directly harm other people. (VANDU, 2010)

From its pragmatic roots, harm reduction also encompasses more of a public health approach, where neutrality toward risky behaviours is based more on pragmatic considerations of best possible health and social outcomes (Klein, 2015). In contrast to theories of addiction that emphasize substance use as a moral failing deserving of punishment or as a disease requiring treatment, harm reduction accepts that some people will continue to use addictive substances and engage in other higher-risk behaviours and sets out to reduce associated harms (Marlatt, 1996). More recently, harm reduction theory has started to examine how harms are structured by broader social, economic, and cultural factors such as income inequality or housing policies (Boucher et al., 2017). Our study is curious about how harm reduction is defined and operationalized in post-secondary settings and considers the full range of harms experienced on campus, from substance use to mental health issues and suicide, sexual violence, harmful power relations, and academic stress.

According to the National College Health Assessment (NCHA), self-reported rates of mental health issues, substance use and sexual violence among Canadian post-secondary students are high and many have increased dramatically between 2013 and 2019 (American College Health Association, 2013, 2016, 2019). While
$12 \%$ of students reported being diagnosed or treated for anxiety disorders over the past 12 months in 2013, this prevalence rate increased to $18 \%$ in 2016 and further increased to $24 \%$ in 2019 . Over the same time period, the number of students surveyed who reported that they considered suicide in the past 12 months also increased from $10 \%$ to $16 \%$ and reported rates of sexual touching and sexual penetration without consent doubled.

Rates of substance use reported by NCHA respondents have not increased since 2013: $63 \%$ of students reported using alcohol and $25 \%$ reported using cannabis in the past 30 days. These rates are similar to those in the general adult population, which suggests that substance use is a commonplace, pleasurable, and unproblematic activity for most people (Centre for Addiction and Mental Health, 2020). However, other NCHA findings are more concerning: $29 \%$ of students report having had five or more drinks in one sitting at least once in the past two weeks, and $12 \%$ of students report having used prescription drugs such as painkillers and stimulants for purposes other than what they were prescribed for in the past year. Recent Canadian and international surveys of younger teenagers are showing a decline in rates of substance use, but to date these shifts have neither persisted into young adulthood nor into post-secondary settings (Canadian Centre on Substance Use and Addiction, 2017; Livingston, 2014).

The extent to which reported increases in sexual violence and mental health issues reflect societal changes as opposed to actual increases in prevalence rates is unclear. Public discussion may be influencing student willingness to report sexual violence, and declining stigma may be contributing to an increased willingness to report mental health issues and to seek help. A similar potential measurement problem can be seen in dramatic increases in emergency department visits and hospitalizations for children and youth with mental health issues over the past decade, despite no known increases in the prevalence of mental health disorders in national health survey data for this population (Canadian Institute for Health Information, 2019). Regardless of the reason, worries about mental illness, substance use, and sexual violence on campus have grown. Canadian post-secondary institutions are reporting surging demands for mental health services and have stated a need for creative solutions (Colleges Ontario et al., 2017; Dixon, 2018).

Post-secondary institutions in Canada have re- 
sponded to these pressures in a variety of ways, from increasing the capacity of campus-based counselling services to launching broad harm reduction and mental health promotion initiatives. Initiatives such as BeWell at Queen's University (2019) and UBC Wellbeing at the University of British Columbia (2019) touch on everything from stress and nutrition to suicide prevention to providing sexual health resources through safer sex initiatives. The Umbrella Project at Algonquin College (2019) is a leading example of a comprehensive harm reduction program focused on substance use. Through training, workshops, education, awareness, and support for students and staff, it seeks to create a safer space on campus to foster open dialogue on the use of drugs and alcohol and the potential harms.

Post-secondary institutions have also taken aim at harms related to binge drinking at parties, both through broad harm reduction programs such as Party in the Right Spirit at the University of Toronto (2019) and Safer Partying at McGill University (Healthy McGill, 2018) and in the context of specific campus events such as frosh week, a week-long orientation for incoming students consisting of various events and parties. Efforts to reduce harms during frosh week range from offering alternatives to banning substance use altogether. McGill University puts on many workshops for new students on harm reduction and sexual consent during orientation week, and offers an array of alternative froshes, such as a rad frosh that focuses on social and political issues, and various religious froshes that help students to build social networks in their new setting (O'Neill et al., 2016). Dalhousie University and Queen's University both instituted dry froshes in response to an alcohol-related death (Maclean's, 2011; Patil, 2017). Many campuses have also begun to offer naloxone training and to provide kits to reduce the risk of death from opioid overdose (Canadian Press, 2017).

Students have a leadership role in most of these harm reduction and mental health promotion programs, from developing new programs to offering training and providing peer support. Student activists are also calling for increased support for harm reduction programming that is explicitly non-judgemental and centred on student empowerment. For example, the Canadian Students for Sensible Drug Policy (2019) advocates for education that "acknowledge[s] and respect[s] young people's personal experience, a discussion that should be approached only in a space of compassion and non-judgment" (para. 4).
Counselling services on campus have tended to focus more on mental health issues and to refer students to services off-campus for substance use problems. McGill University's Counselling Services has been noteworthy for its adoption of Tatarsky and Marlatt's (2010) integrative harm reduction psychotherapy, a strengthsbased approach that meets students where they are at and is focused on empowerment and collaboration. At the time of this study, McGill University was in the process of launching a new centralized wellness hub, although the role of harm reduction in counselling services offered through this new hub was not yet clear (McGill University, 2019).

The development of a new Canadian Standard on Psychological Health and Safety for Postsecondary Students is unfolding against this backdrop of increased attention to mental health and substance use-related harms in post-secondary settings (Mental Health Commission of Canada, 2019). This post-secondary standard is modelled after the National Standard of Canada for Psychological Health and Safety in the Workplace (CSA Group et al., 2014). With a view to increasing employee satisfaction and productivity and reducing legal liability, the workplace standard has a strong emphasis on reducing harms that are inherent to workplace settings. Examples of such workplace-specific harms include harassment, lack of recognition and influence over one's work, and overwhelming job demands. In keeping with this model, it is important to also consider harms inherent in post-secondary environment in the development of a post-secondary mental health standard, in addition to the importance of better clinical services and mental health promotion programming.

Academic researchers have also been responding to these growing concerns about mental health and substance use-related harms on campus. An emerging field of literature regarding harm reduction within the post-secondary context has focused mainly on substance use, primarily alcohol, and secondarily on illegal drug use. This research has identified post-secondary settings as unique risk environments for substance-use related harms. Further, in tracking and evaluating everything from harm reduction to zero tolerance approaches, research is illuminating divisions and inconsistencies in how harm reduction is defined and operationalized on campus.

Risk environments are settings in which overlapping factors increase vulnerability to harm stemming 
from substance use (Rhodes, 2009). Such overlapping social, cultural, and environmental factors within the post-secondary setting include the independence from guardians, the interconnectedness of students, and the normalization of substance use (Moyle \& Coomber, 2019). Substance use is often linked to the university experience, where alcohol is readily available and a party culture promotes excessive drinking and drug experimentation (Wilkinson \& Ivsins, 2017; Moyle \& Coomber, 2019). Harms associated with substance use include health problems, academic issues, and physical and sexual violence (Snow et al., 2003; Villarosa et al., 2018). Alcohol is perceived as a tool for fostering social bonds to such an extent that abstinence can be socially isolating (Brown \& Murphy, 2018; Snow et al., 2003). Students who do not drink are also exposed to risk through the second-hand effects of excessive substance use, including interpersonal conflict, property damage, and physical and sexual victimization (Wilkinson \& Ivsins, 2017). The post-secondary experience can also be associated with an increased risk of mental health issues, which can in turn be compounded by increased risk or problematic substance use (Kazemi et al., 2014; Shaffer et al., 2005; Villarosa et al., 2018; Wilkinson \& Ivsins, 2017). Another factor in this unique risk environment is that demanding schedules and the normalization of substance use can lead students to be reluctant to seek help (Shaffer et al., 2005; Snow et al., 2003).

Researchers have been tracking and evaluating the wide range of official and unofficial strategies being implemented to mitigate the elevated risk of harms in post-secondary settings in countries such as Canada, the United States, Australia, New Zealand, and the United Kingdom. Preventative measures range from alcohol education programs and advertising restrictions to alcohol free-residences, dry events, and policy reforms (Shaffer et al., 2005; Wilkinson \& Ivsins, 2017). There is also a divide between punitive, prohibitionist policies as opposed to more non-judgemental approaches that are more in keeping with the pragmatic and activist principles associated with harm reduction. This divide tends to align with broad societal values. For example, while prohibitionist approaches that ban underage drinking on campus are in place at almost all campuses in the United States, post-secondary substance use policies in Australia are influenced by a harm minimization policy that was adopted in 1985 (Australian Government, 2004;
Shaffer et al., 2005). Students have been found to prefer harm reduction approaches such as alcohol-alternatives and responsible alcohol service to more punitive measures (Snow et al., 2003). Relative to zero-tolerance messaging, harm reduction approaches may be particularly effective with students who are already drinking (Napper, 2019). However, harm reduction approaches that do not take the pleasure derived from substance use into account have fallen flat, particularly with students who choose to drink five or more drinks on one occasion (Hutton, 2012).

Regardless of the approach taken and as evidenced by the NCHA data cited above, substance use-related problems remain prevalent across post-secondary campuses (American College Health Association, 2019). Prohibitionist policies have failed to reduce substance use for many populations, and further have a disproportionately negative effect on students suffering from underlying mental health issues (Abelman, 2017; Wilkinson \& Ivsins, 2017). Harm reduction approaches have also had little impact on student consumption, perhaps because students dismiss tips on a safe night out and warnings about risks as paternalistic (Brown \& Murphy, 2018).

Within post-secondary institutions, researchers have found inconsistencies in how harm reduction is defined and operationalized that echo the deep divide between more punitive and more non-judgemental approaches. Among administrators, staff, and students, some believe that the harms associated with alcohol are inconsequential and others believe they are underestimated (Snow et al., 2003). In Brown and Murphy's (2018) study, some staff members expressed that they did not believe harm reduction solutions would be effective but that universities nevertheless provide such tips for safer substance use out of a sense of duty. The wide range of views held by post-secondary stakeholders make it difficult to formulate a standardized policy, and to enforce and evaluate such policies (Snow et al., 2003; Wilkinson \& Ivsins, 2017). In light of these challenges, researchers have been encouraging policy reform based on evidence and best practices. Harm reduction measures have been found to be most effective when they address underlying risk factors such as mental health issues and low self-esteem, when they are created alongside students, and when they reflect the unique post-secondary culture and setting (Abelman, 2017; Wilkinson \& Ivsins, 2017). 
From this review of post-secondary reforms and the academic literature, it is clear that harm reduction is at once an emerging policy priority and a highly contested concept in post-secondary settings. This study builds on and contributes to this literature by building a deep understanding of challenges and opportunities for a more evidence-based and consistent approach to harm reduction, from the perspective of student leaders, student service providers, and administrators. This study also makes a contribution by examining how the focus of harm reduction is being broadened beyond substance use in post-secondary settings. The results can strengthen the extent to which initiatives such as the Standard on Psychological Health and Safety for Post-Secondary Students focus not just on mental health promotion and counselling services, but also on the broad range of harms that are inherent to post-secondary settings (Mental Health Commission of Canada, 2019).

\section{Methodology and Methods}

This qualitative case study is based on interpretive phenomenology, whereby researchers interpret the perspectives of people with deep lived experience of a particular phenomenon (Neubauer et al., 2019; Smith, 2011). To elicit deep lived experiences of the challenges and opportunities associated with harm reduction in post-secondary settings, we held separate focus groups with student leaders, service providers, and administrators at McGill University. Student leaders held leadership roles in diverse health promotion services on campus, service providers provided a range of university student services, and administrators held leadership roles in student services. To promote the transparency and overall quality, our study was guided by the 32-item Consolidated Criteria for Reporting Qualitative Research (COREQ) checklist designed to systematize qualitative research (Tong et al., 2007). The COREQ includes guiding questions on research team and reflexivity, study design, and data analysis, and fostered in-depth reflection regarding each stage of the research process. The study design was reviewed and approved by McGill University's Research Ethics Board, and all participants provided informed consent.

McGill University, a large and prestigious Canadian university, was selected for the study design as it has a history of strong harm reduction programming. Sepa- rate focus groups were held for student leaders, service providers, and administrators to promote free and open discussion amongst groups with different levels of power within post-secondary settings. Using a purposive sampling approach, participants were recruited based on a scan of McGill's harm reduction structures and services. Out of 23 invitees, a total of 17 participants took part in the study, including seven students, four service providers, and six administrators. The three focus groups were all held in person at McGill University in March 2019, with each group lasting between 1.5 and 2 hours. Participants were asked two broad questions: what are the challenges related to harm reduction in post-secondary settings and what are the opportunities? Through a mix of group discussion, discussion in pairs, and individual reflection sheets, participants shared their perspectives. Data from the focus groups was captured through transcriptions of audio recordings that captured the discussion verbatim, real-time notetaking that added a layer of synthesis from the perspective of the research team, and reflection sheets that provided participants with an opportunity to add additional ideas that we did not have time to discuss. In order to maintain confidentiality, quotes are attributed to participants identified by type and number only. For example, a student leader could be identified as SL_1, a service provider as SP_4, and an administrator as A_2 and so forth.

The focus groups were facilitated and analyzed by a research team composed of two interns from the Institute of Health and Social Policy under the supervision of a postdoctoral researcher with expertise in mental health and substance use policy. All researchers identify as women. The interns had previous experience working as student service leaders at McGill University, one within the context of mental health and addictions, and the other regarding sexual health and sexual violence.

In keeping with an interpretive phenomenological approach, this study reveals recurrent themes across the focus groups by presenting the participant voices through quotes and the researchers' insights through analysis. The research team originally identified 19 themes from the focus groups addressing challenges such as turnover, party culture, and bureaucracy, as well as opportunities such as social relations, community care, and motivation for change. Data analysis was conducted through detailed line-by-line coding of the transcripts using Nvivo 12 software. A portion of each focus group 
Table 1

Harm Reduction in Postsecondary Settings: Summary of Key Themes

\begin{tabular}{ll}
\hline Key Themes & Illustrative Quotes \\
Work hard, party hard & $\begin{array}{l}\text { In academic culture, students tend to glorify being absolutely wrecked. How } \\
\text { could harm reduction undo that kind of glorification? (Student Leader) }\end{array}$ \\
[A security guard] acting on what societal norms would be saying I need to \\
take that [bag of cocaine] away from you vs a floor fellow feeling that anything \\
related to any kind of shame needs to be avoided. (Administrator)
\end{tabular}

transcript was coded by all three researchers and then cross-validation was conducted as the initial coding was carried out. This long list of themes was then condensed into the four themes reported below, and cross-validated again to ensure consistency and clarity. The findings were further validated through feedback from harm reduction experts and student service leaders, including at a workshop at the national conference of the Canadian Association of College and University Student Services.

\section{Results}

The research team identified four key themes that cut across participants' experiences surrounding harm reduction in post-secondary settings: (a) a work hard, party hard culture where students attempt to balance schoolwork and social life, (b) a clash of the titans between opposing harm reduction approaches, (c) broad approaches to harm reduction that extend far beyond substance use but also risk being applied as band-aid solutions that do not address root causes, and (d) knowledge and priv- ilege as unique features of an academic environment. Illustrative quotes can be found in Table 1 .

\section{Work Hard, Party Hard Culture}

Students, service providers, and administrators consistently voiced that a work hard, party hard culture is prevalent within post-secondary settings (perhaps even more so than most at McGill University) and poses risks. Participants stressed that academic pressures paired with practices of excessive substance use can be a harmful combination. This work hard, party hard culture was linked to social standing, pedagogical practices, and the normalization of substance use.

Participants framed substance use as interconnected with social bonding and career advancement in the post-secondary context. According to these service providers, "the culture is the work hard, party hard culture and taking that out of the equation, it's part of the identity, the experience at McGill" (SP_1) and "in the university setting, there are large drinking events that occur that 
play into somebody's social status and connection with the community" (SP_2). Drinking was linked not only to social acceptance, but also potential academic and career connections to the point that not drinking could be limiting. As one administrator stated:

Networking often means drinking, or going to parties often means there are going to be substances... We have heard from different faculties, if you don't drink, you're out. Does that mean you're out of career opportunities, does that mean you're out of social circles? (A_3)

Further, participants identified pedagogical practices as a potential harm within the post-secondary setting that can place undue burdens on students and may also contribute to substance use. According to these student leaders, "There is an opportunity to look at harm reduction in academic context, at how to design assignments and syllabi without placing undue stress on student mental health" (SL_1) and "There is an opportunity to apply harm reduction to classroom pedagogy. Currently, the way tutorials and conferences are facilitated, the existence of $85 \%$ finals in math, are stressful and not good pedagogy" (SL_4). Due to such academic pressures to work hard, students used substances to de-stress from academic pressure by partying hard. A service provider described this dynamic as follows: "They are coming in because they are so stressed out and anxious, and by the way 'I smoke pot every day,' or 'I get blackout drunk every weekend"' (SP_4). Student leaders also pointed out how students attempt to enhance academic performance with study drugs: "In academic culture, students tend to glorify being absolutely wrecked, popping Adderalls" (SL_1).

Moreover, this work hard, party hard culture becomes normalized and can function to conceal substance use problems. Service providers highlighted how being in settings where many people use excessively can affect students' perception of their own usage. For example:

If you're in a peer group that uses heavily you are going to end up thinking your usage is normative compared to your group. Everyone always has the one friend Johnny who does more than me, I don't have a problem, Johnny does $x y z$. It's always in that kind of comparison, that is where it becomes oftentimes tricky for people to self-identify that there is a strug-

\section{gle. (SP_4)}

While participants mentioned efforts toward changing this work hard, party hard culture, such attempts were viewed as limited by the structural and social context of post-secondary institutions that encourage events that involve excessive drinking such as frosh. As one student asked, "what do we do about parts of [the post-secondary experience] that are inherently harmful, events that are inherently harmful like frosh? We can't harm reduce this" (SL_4). In the administrators' focus group, participants described how students themselves are changing their attitudes and expectations. For example:

A lot of our students don't want to engage with a harm reduction approach at all. They would actually prefer a more "no, abstinence is the way."... We see that in the changing culture around frosh and idea of changing the perception of what it means to be a new student at the university. (A_4)

However, a student leader stated that culture change on campus is ultimately limited by the endurance of the work hard, party hard expectation in the broader society: "Even if everyone were getting peer education, the outside world still tells people that on a Friday night you go party with your friends, then you stay up all night studying" (SL_3).

\section{A Clash of the Titans}

Tensions between how harm reduction is interpreted and applied were identified as a central challenge by focus group participants, with students taking an activist, non-judgemental approach, administrators balancing concerns over risk and liability with pragmatic considerations regarding the need for harm reduction approaches, and security personnel called in to prevent immediate harm in what are often crisis situations. One administrator described these tensions as a clash of the titans, where students adopt something akin to an "aggressively value-neutral stance" to harm reduction (Klein, 2015, p. 465) and where security officers may not be versed in harm reduction principles:

This becomes a clash of the titans of what is harm reduction... a zealous group [students] that are advocates for all levels, extreme levels of harm reduction 
vs. another group [security officers] that may be less informed. So dealing specifically, for example, with possession of a drug: what is the proper way to address that? Both sitting at extreme ends and not necessarily being in line with where the university wants to be. (A_5)

One of the sites in which this "clash of the titans" was most evident was within residences, where students form their own networks of support and harm reduction strategies with support from floor fellows (experienced students who act as advisors and live in student residences). As one student leader explained:

For floor fellows, harm reducing interactions are about [being] available for first year students.... They came in to talk about drugs and alcohol, for example, they could say, "I am going to take acid for the first time, do you mind if I do it here? Can you check in on me?" (SL_5)

While administrators noted that harm reduction policies in residences were "quite nuanced and very strong" (A_4), they also expressed concerns that some students take the non-judgemental aspects of harm reduction too far. Moreover, the divisions between the perspectives of students and first responders can exacerbate tensions during crisis situations, as can be seen in the following quotes: "[A security guard] acting on what societal norms would be, saying 'I need to take that [bag of cocaine] away from you,' versus a floor fellow feeling that anything related to any kind of shame needs to be avoided" (A_2); "[sometimes] floor fellows get in front of first responders correcting their behaviour, to tell them you shouldn't be speaking to a student that way. The most recent example was for a suicide attempt" (A_5); and "Some parts of the community would never want to call 911 because there is a philosophy around reducing harm and a belief that the first responders in fire truck are actually going to cause more harm" (A_4).

Participants highlighted how legal complexities and institutional responsibilities underpin tensions regarding harm reduction on campus. For example:

Institutional risk is a huge issue. You could make arguments on various parts of it. The risk of adopting an approach where there is a high degree of tolerance for certain substances within a residence community is itself risky, but there's also risk on the other side. Flare ups...becomes quite political and quite a challenging discussion institutionally. (A_4)

Moreover, participants questioned whether there is any possibility of resolving such tensions given these institutional constraints. One student leader asked: "What are the dynamics with harm reduction as a political statement, particularly within a university administration that does not like political statements?" (SL_3). Similarly, a service provider identified the following as a key challenge: "Divided opinions in higher administration on whether or not topics like harm reduction should be directly addressed" (SP_3).

\section{Broad Approaches and Band-Aid Solutions}

Student leaders are reconceptualizing harm reduction in new and more critical ways, both as something newly broad and as something that risks being used as a bandaid solution to cover over underlying issues. As has been evident in the discussion of the two previous themes, participants identified how harm reduction is being applied by students to a far broader range of topics than are typically associated with the concept. This breadth can be seen in these quotes from a student leader and an administrator: "The language of harm reduction gets used to cover lots and lots of things, not just substance use" (SL_3) and "For us mental health professionals, we have a very specific idea of what we mean by [harm reduction]. But then when it is community work, I was surprised how rogue and broader it seemed to go" (A_1).

Such areas not only include substance use but also mental health and well-being, sexual health and sexual violence, suicide prevention, academic pedagogy, and social relations. At first glance, it may seem inappropriate or even offensive to use harm reduction in such cases. How can one take a harm reduction approach, which does not require stopping a behaviour, to unacceptable behaviours such as sexual violence, racism, or emotional abuse? However, students' broad approach of harm reduction seems to come from a recognition that harms in post-secondary settings are likely to continue and can thus benefit from harm reduction approaches. For example, with regard to sexual violence: "Our [active bystander] workshops have a clear harm reduction role, 
specifically with the aim of [sexual violence] prevention in a high-risk space" (SL_6). With regard to harmful social relations, student leaders raised questions regarding race and gender as well as the powerlessness students can experience in their relationships with professors. According to one student leader: "Another important area for harm reduction is supervisor on student emotional abuse. When this happens, students can be stuck in that harmful relationship for five years, what do you do without killing your career?" (SL_7).

Even as they staked out a new, broader understanding of harm reduction, student leaders also expressed concerns that harm reduction and mental health initiatives were being used as band-aid solutions that do not address underlying root causes. According to one student leader:

With mental health, how much are we dealing with having to stay up all night to pass an exam as opposed to [the university] providing therapy dogs and recommending that people get some exercise or "go for a walk on the mountain." (SL_4)

Both student leaders and service providers described how high turnover and limited resources make it hard to address issues on a deeper level. For example:

Turnover at the staff/student leader level gets in the way of substantial change, and ties into resistance of general system change. Student leaders have different priorities so it is hard to get momentum as people are elected in and out. Governance and institutional priorities are also challenges. The constant in and out of the population makes it challenging. (SL_5)

Further, "because we are a short-term service, when people require longer term support, we don't necessarily always have a place to send them, especially if there is a co-occurring difficulty like substance use and sexual violence" (SP_2). Student leaders also talked about how some groups such as fraternities ("frats") on campus invoke harm reduction as a framework in order to keep up appearances and the challenges in calling out such performative behaviours: "Social capital is associated with certain words such as harm reduction. For example, a frat attending a workshop on responding to disclosure [of sexual violence] insulates them from criticism" (SL_4) and "You can't...call out performative use of workshops, without institutional support" (SL_6). Lastly, harm reduction can sometimes be misused to minimize behaviours that should not be reduced but condemned, such as sexual violence: "There is an opportunity to recognize that the fact that sexual violence is wrong is not negotiable. And to make training mandatory early on, for example for varsity teams" (SL_3).

\section{Knowledge Transfer and Privilege as Unique Features}

The final theme focused on how harm reduction is shaped by two unique features of post-secondary settings: knowledge transfer and privilege. First, a common theme across all three focus groups was the unique opportunity for knowledge transfer about harm reduction in post-secondary settings, through education, research, collaboration, and hiring practices. With regard to education, one service provider explained the unique opportunities for harm reduction at this stage of life: "Because they are emerging adults, it's a good population for trying different health promotion strategies. They are gaining this autonomy, but also there is a certain resilience and possibility for growth in this population" (SP_3). Participants also highlighted how having students as a captive audience created ample opportunities for awareness and outreach across campus during events, orientation, and in grassroots organizing.

Post-secondary settings were also described as centres where knowledge about harm reduction can be produced and disseminated:

One thing that can obviously occur [here] is that interested faculty that are studying these kinds of subjects, can transfer this information both to the campus and to the wider public. There can be knowledge transfer between researchers and health promotion, there can be knowledge transfer between researchers and senior administration, there can be many different arrows you could draw. (SP_2)

Administrators spoke about the ample opportunity for collaboration and for building on existing knowledge:

Usually most of the focus groups or committees that I have participated in, everyone is willing to help, to participate and share.... The knowledge that we have as well, all of these years of experience and examples 
as well that we've gone through and learned from, that we have kind of adapted and modified. (A_2)

One additional opportunity is the collaborations, including different voices within the institution. Whenever there is a task force such as the task force for cannabis, it is really great, that was an opportunity for bringing different student voices, administration, legal, those kinds of spaces. (A_1)

This same knowledge base was also identified as an opportunity to guide hiring practices: "We have many case studies to draw from; [we can] be purposeful when hiring staff and align harm reduction concepts into hiring practices" (A_4).

Second, participants also described the unique opportunities and challenges regarding harm reduction within a post-secondary setting due to the privileged position of post-secondary students in society as a whole, particularly in an elite institution such as McGill University. According to one administrator: "Positionality is a huge component to look at, when looking at our audience. The level of privilege is different in terms of looking at the application of harm reduction in a post-secondary environment versus the society at large" (A_5). Student leaders also acknowledged how they can wield privilege and can even perpetuate harm. For example, one student stated: "Universities don't exist in vacuums. By virtue of being at universities, students can be perpetrators of harm for example through gentrification and displacing communities" (SL_1). Some students hold more privilege than others and certain subgroups were identified as being additionally vulnerable within the post-secondary environment. Participants raised concerns over how first-generation students, students who have criminal records, and international students faced additional barriers in navigating resources and fell through gaps in support services. One service provider explained how eligibility for accommodations is dependent on access to documentation:

People with access to better psychologists or more psychologists, more healthcare, will get that proper documentation. It is not easy to get that documentation. But if you're a first-generation university student or just don't know, you're out of luck so to speak. (SP_3)

\section{Discussion}

The themes identified in this phenomenological case study bring out deep knowledge of the unique challenges and opportunities for harm reduction in one post-secondary setting and contributes to the knowledge regarding harm reduction more broadly. While student leaders, administrators, and service providers brought different perspectives and experiences of harm reduction at McGill University, the frank and open discussions in each of the three focus groups touched on similar themes. Most notable among the challenges is a pervasive work hard, party hard culture that underscores the importance of not just addressing social norms regarding substance use on campus, but also the interplay with norms regarding the drive for academic excellence. Other challenges identified include clashes between student activists and institutional interests of the university regarding how to define and operationalize harm reduction, and band-aid solutions that are not able to get at underlying issues. Interesting opportunities arise from the way that students are broadening the definition of harm reduction to apply to many risks that are endemic in post-secondary settings, including substance use but also mental health, sexual violence, and social relations. The academic context creates opportunities for knowledge transfer and collaboration, and the relative privilege of post-secondary students creates both opportunities and challenges as such privilege is not equitably distributed across the student population. In keeping with the activist roots and more recent structuralist approach to harm reduction, this study points to the importance of including students in the design of harm reduction approaches on campus in a way that considers broader structural inequities within the student population.

This qualitative case study has several limitations associated with a phenomenological approach. While our three focus groups provide deep insight into one large Canadian university, further research would be needed to determine if our findings are generalizable to smaller post-secondary settings or to less academic settings. The culture and norms in post-secondary institutions may also be different in Canadian provinces other than Quebec, which is known to have a more liberal attitude toward alcohol, and in other countries that have their own unique socio-economic and cultural contexts surrounding substance use (Measham, 2006). The decision to host three separate focus groups did foster open discussion 
among participants with similar levels of power, but also limited exploration of opportunities for collaborative approaches to harm reduction between student leaders, service providers, and administrators. Additional perspectives from individuals not directly involved in the provision of harm-reduction programming and policies would also provide important information on challenges and opportunities. Further, while the empirical findings in this study raise important theoretical questions about the tensions between activist, pragmatist, and security-oriented approaches to harm in post-secondary settings, a more theoretical and critical analysis is needed to fully explore the theoretical implications.

At the same time, a phenomenological case study provided nuanced insights from participants with diverse perspectives, positionalities, and experience with harm reduction in a post-secondary setting. As an interpretive phenomenological study, our analysis was further strengthened by a research team with its own deep and diverse knowledge of harm reduction in post-secondary settings, and our ability to cross-validate our analysis with each other and through feedback from the student services community helped to reduce the risk of bias.

The findings suggest possible avenues for harm reduction approaches in post-secondary settings that could be implemented as part of the new Standard for Psychological Health and Safety of Postsecondary Students (Mental Health Commission of Canada, 2019). Much as the National Standard of Canada for Psychological Health and Safety in the Workplace has focused on reducing harms that are inherent to workplace settings, there is much that universities and colleges can do to reduce harms that are inherent to post-secondary settings such as binge drinking, sexual violence, and academic stress. Work hard, party hard culture can be addressed through policy reforms that go beyond band-aid solutions, including student-centred pedagogical practices, alternatives to drinking-focused events such as frosh, and mandatory sexual violence prevention training, in addition to increased resources for counselling services and wellness programming. While the concentration of young people away from home for the first time contributes to what Rhodes (2009) called risk environments, post-secondary institutions can also capitalize on their unique opportunity to reach a somewhat captive audience early in adulthood. Further, post-secondary settings are in a unique position to leverage the relative privilege of post-secondary students as well as opportunities for research and collaboration.

The findings also point to ways in which harm reduction in post-secondary settings could open up avenues for harm reduction in society as a whole. The clash of the titans between the activist harm reduction approach of student leaders, the pragmatist orientation of administrators, and the law and order starting point of security personnel is a microcosm of broader societal divisions regarding harm reduction. Attempts to address these divisions head on could be counterproductive, as increased attention could lead post-secondary institutions to shift the balance away from pragmatism and toward risk management. Nevertheless, if these groups could work together to develop scenarios for training purposes, such collaborative efforts might not only reduce tension during crisis situations on campus but could also have practical applications outside of post-secondary settings. To be successful, such collaborative efforts require levelling power imbalances as much as possible to ensure that student voices are heard, particularly the voices of students with less privileged positions in post-secondary settings and in society. Looking to the future, the broad approach to harm reduction taken by student leaders may pave the way for a less divisive conceptualization of harm reduction in the future. As this cohort matures and takes up leadership positions in society, harm reduction may have less to do with debates over illegal drugs and abstinence, and more to do with wellness, inclusion, and a rejection of shame.

\section{Conclusion}

In the context of an increasing focus on psychological health and safety in post-secondary settings, this qualitative study explores the challenges and opportunities for harm reduction through focus groups with student leaders, service providers, and administrators in one large Canadian university. Key themes explored by participants include a pervasive work hard, party hard culture, clashes regarding how to define and operationalize harm reduction, a broadening definition of harm reduction and risks of band-aid solutions, and knowledge transfer and privilege in an academic context. These findings suggest possible avenues for harm reduction that could be implemented as part of the new post-secondary standard, as well as in society as a whole. 


\section{Acknowledgements}

The authors would like to thank the participants in this study, and to acknowledge the financial support of the Institute for Health and Social Policy at McGill University and the Canadian Institutes for Health Research (G245655 CIHR PJT-153012).

\section{References}

Abelman, D. D. (2017). Mitigating risks of students use of study drugs through understanding motivations for use and applying harm reduction theory: A literature review. Harm Reduction Journal, 14(68). https:/l doi.org/10.1186/s12954-017-0194-6

Algonquin College. (2019). Umbrella Project. https:ll www.algonquincollege.com/umbrellaproject/

American College Health Association. (2013). American College Health Association-National college health assessment II: Canadian reference group data report spring 2013. American College Health Association.

American College Health Association. (2016). American College Health Association-National college health assessment II: Canadian reference group data report spring 2016. American College Health Association.

American College Health Association. (2019). American College Health Association-National college health assessment II: Canadian reference group data report spring 2019. American College Health Association.

Australian Government. (2004). Module 9: Working with young people on AOD issues: Learner's workbook, 2.1 harm minimization. https://www1.health.gov.aul internet/publications/publishing.nsf/Content/drugtreat-pubs-front9-wk-toc drugtreat-pubs-front9-wksecb drugtreat-pubs-front9-wk-secb-2 drugtreatpubs-front9-wk-secb-2-1

Boucher, L. M., Marshall, Z., Martin, A. L., LaroseHébert, K., Flynn, J. V., Lalonde, C., Pineau, D., Bigelow, J., Rose, T., Chase, R., Boyd, R., Tyndall, M., \& Kendall, C. (2017). Expanding conceptualizations of harm reduction: Results from a qualitative community-based participatory research study with people who inject drugs. Harm Reduction Journal, 14(18), 1-18. https://doi.org/10.1186/s12954-017$\underline{0145-2}$

Brown, R., \& Murphy, S. (2018). Alcohol and social connectedness for new residential university students: Implications for alcohol harm reduction. Journal of Further and Higher Education, 44(2), 216-230. https://doi.org/10.1080/0309877X.2018.1527024

Canadian Centre on Substance Use and Addiction. (2017). Canadian Drug Summary - Alcohol. https:/l www.ccsa.ca/sites/default/files/2019-04/CCSA-Canadian-Drug-Summary-Alcohol-2017-en.pdf

Canadian Students for Sensible Drug Policy. (2019). Canadian Students for Sensible Drug Policy chapter (University of Toronto). https://ulife.utoronto.cal organizations/view/id/2638

Canadian Press. (2017, September 6). Canadian universities training staff to deal with opioid overdoses. Maclean's. https://www.macleans.ca/news/ canadian-universities-training-staff-to-deal-with-opioid-overdoses/

Canadian Institute for Health Information. (2019). Child and youth mental health in Canada. https://www. cihi.ca/en/child-and-youth-mental-health-in-canadainfographic

Centre for Addiction and Mental Health. (2020). Understanding substance use problems and addiction: Reasons for substance use. https://moodle8.camhx. $\mathrm{ca} / \mathrm{moodle} / \mathrm{mod} / \mathrm{book} / \mathrm{view}$.php?id=113\&chapter$\underline{i d}=211$

Colleges Ontario, the Council of Ontario Universities, the College Student Alliance, \& the Ontario Undergraduate Student Alliance. (2017). In it together: Taking action on student mental health. https://docs.wixstatic.com/ugd/0b1b3b c6a06fcd038d4e90a1dbd29def48a889.pdf

CSA Group, Bureau de normalisation du Québec, Standards Council of Canada, \& Mental Health Commission of Canada. (2014). Psychological health and safety in the workplace: prevention, promotion, and guidance to staged implementation. http://www. deslibris.ca/ID/241415 
Dixon, G. (2018, October 31). Growing mental-health needs of students require creative solutions. The Globe and Mail. https://www.theglobeandmail.com/ canada/education/canadian-university-report/articlegrowing-mental-health-needs-of-students-requirecreative-solutions/

Government of Canada \& Health Canada. (2018). Background document: Public consultation on strengthening Canada's approach to substance use issues. http://publications.gc.ca/collections/collection 2018/ sc-hc/H14-266-2018-eng.pdf

Hawk, M., Coulter, R. W. S., Egan, J. E., Fisk, S., Reuel Friedman, M., Tula, M., \& Kinsky, S. (2017). Harm reduction principles for healthcare settings. Harm Reduction Journal, 14(70). https://doi.org/10.1186/ s12954-017-0196-4

Healthy McGill. (2018). Safer partying 101 [webinar]. https://mcgill-stuserv.adobeconnect.com/ phv9hyiidows/?launcher=false\&csContent=true\&pbMode=normal

Hutton, F. (2012). Harm reduction, students and pleasure: An examination of student responses to a binge drinking campaign. International Journal of Drug Policy, 23(3), 229-235. https://doi. org/10.1016/j.drugpo.2011.10.001

Kazemi. D. M., Cochrane, A. R., Kelly, J. F., Cornelius, J. B., \& Belk, C. (2014). Integrating mHealth mobile applications to reduce high risk drinking among underage students. Health Education Journal, 73(3), 262-273. https://doi. org/10.1177/0017896912471044

Klein, A. (2015). Criminal law and the counter-hegemonic potential of harm reduction. Dalhousie Law Journal, 38(2), 2-27.

Livingston, M. (2014). Trends in non-drinking among Australian adolescents. Addiction Research Report, 109(6), 922-929. https://doi.org/10.1111/add.12524

Maclean's. (2011, July 27). Queen's to ban alcohol during Frosh Week. https://www.macleans.ca/education/uniandcollege/queens-to-ban-alcohol-duringfrosh-week/

Marlatt, G. A. (1996). Harm reduction: Come as you are. Addictive Behaviors, 21(6), 779-788. https://doi.

\section{org/10.1016/0306-4603(96)00042-1}

McGill University. (2019). Student wellness hub. https:// mcgill.ca/wellness-hub/

Measham, F. (2006). The new policy mix: Alcohol, harm minimisation, and determined drunkenness in contemporary society. International Journal of Drug Policy, 17(4), 258-268. https://doi.org/10.1016/j. drugpo.2006.02.013

Mental Health Commission of Canada. (2019). Postsecondary students standard. https://www.mentalhealthcommission.ca/English/studentstandard

Moyle, L., \& Coomber, R. (2019). Student transitions into drug supply: Exploring the university as a "risk environment." Journal of Youth Studies, 22(5), 642-657. https://doi.org/10.1080/13676261.2018.1529863

Napper, L. E. (2019). Harm-reduction and zero-tolerance maternal messaged about college alcohol use. Addictive Behaviors, 89, 136-142. https://doi. org/10.1016/j.addbeh.2018.09.024

Neubauer, B. E., Witkop, C. T., \& Varpio, L. (2019). How phenomenology can help us learn from the experiences of others. Perspectives on Medical Education, 8(2), 90-97. https://doi.org/10.1007/ s40037-019-0509-2

O'Neill, E., Rahman, N., Sukhia, R., Kearney-Fick, A., \& Davis, J. (2016, September 20). Campus conversation: How effective is Frosh as an introduction to life at McGill? The McGill Tribune. https://www. mcgilltribune.com/opinion/campus-conversationhow-effective-is-frosh-as-an-introduction-to-life-atmcgill-438749/

Patil, A. (2017, August 30). Dalhousie bans booze during orientation week in "harm-reduction" effort. CBC

News. https://www.cbc.ca/news/canada/nova-scotia/ dal-takes-harm-reduction-approach-to-curb-campusdrinking-1.4267098

Queen's University. (2019). Queen's University be well. https://www.facebook.com/queensubewell/

Rhodes, T. (2009). Risk environments and drug harms: A social science for harm reduction approach. The International Journal on Drug Policy, 20(3), 193-201.

Shaffer, H. J., Donato, A. N., LaBrie, R. A., Kidman, R. C., 
\& LaPlante, D. A. (2005). The epidemiology of college alcohol and gambling policies. Harm Reduction Journal, 2(1). https://doi.org/10.1186/1477-7517-2-1

Smith, J. A. (2011). Evaluating the contribution of interpretative phenomenological analysis. Health Psychology Review, 5(1), 9-27. https://doi.org/10.10 80/17437199.2010.510659

Snow, P. C., Wallace, S., Staiger, P. K., \& Stolz-Grobusch, B. J. (2003). "As long as it doesn't spill over into class": Harms arising from students' alcohol use, and the role of policy in reducing them. International Journal of Drug Policy, 14(1), 5-16. https://doi. org/10.1016/S0955-3959(02)00198-6

Tatarsky, A., \& Marlatt, G. (2010). State of the art in harm reduction psychotherapy: An emerging treatment for substance misuse. Journal of Clinical Psychology, 66(2), 117-22. https://doi.org/10.1002/jclp.20672

Tong, A., Sainsbury, P., \& Craig, J. (2007). Consolidated criteria for reporting qualitative research (COREQ): A 32-item checklist for interviews and focus groups. International Journal for Quality in Health Care, 19(6), 349-57. https://doi.org/10.1093/intahc/ mzm042

University of British Columbia. (2019). UBC wellbeing. https://wellbeing.ubc.cal

University of Toronto. (2019). Safer party tips from party in the right spirit. https://www.utsc.utoronto.ca/hwc/ safer-party-tips-party-right-spirit

Vancouver Area Network of Drug Users. (2010). VANDU manifesto for a drug user liberation movement. http://www.vandu.org/manifesto/

Villarosa, M. C., Messer, M. A., Madson, M. B., \& Zeigler-Hill, V. (2018). Depressive symptoms and drinking outcomes: The mediating role of drinking motives and protective behavioral strategies among college students. Substance Use \& Misuse, 53(1), 143-153. https://doi.org/10.1080/10826084.2017.1 $\underline{327974}$

Wilkinson, B., \& Ivsins, A. (2017). Animal house: University risk environments and the regulation of students' alcohol use. International Journal of Drug Policy, 47, 18-25. https://doi.org/10.1016/j.drugpo.2017.06.002

\section{Contact Information}

Mary Bartram

mary.bartram@carleton.ca 